\title{
ON THE INFINITE TIME HORIZON LINEAR-QUADRATIC REGULATOR PROBLEM UNDER A FRACTIONAL BROWNIAN PERTURBATION
}

\author{
Marina L. Kleptsyna ${ }^{1, *}$, Alain Le Breton ${ }^{2, *}$ and Michel Viot ${ }^{2}$
}

\begin{abstract}
In this paper we solve the basic fractional analogue of the classical infinite time horizon linear-quadratic Gaussian regulator problem. For a completely observable controlled linear system driven by a fractional Brownian motion, we describe explicitely the optimal control policy which minimizes an asymptotic quadratic performance criterion.
\end{abstract}

Mathematics Subject Classification. 60G15, 60G44, 93E20.

Received July 19, 2004.

\section{INTRODUCTION}

Recently, stochastic models appropriate for long-range dependent phenomena have attracted a great deal of interest and numerous theoretical results and successful applications have been already reported. In particular, several contributions in the literature have been devoted to the extension of the classical theory of continuoustime stochastic systems driven by Brownian motions to analogues in which the driving processes are fractional Brownian motions (fBm's for short). The tractability of the standard problems in prediction, parameter estimation and filtering is now rather well understood (see, e.g., $[6-9,15,18,19]$ and references therein).

As far as we know, concerning optimal control problems, it is not yet fully demonstrated. Nevertheless, see [1] for a recent attempt in a general setting and [10] for a complete solution of the simplest linear-quadratic problem on a finite time interval. Here our aim is to illustrate further the actual solvability of control problems by exhibiting an explicit solution for the basic infinite time fractional linear-quadratic regulator problem.

We deal with the fractional analogue of the so-called linear-quadratic Gaussian regulator problem in one dimension. The real-valued state process $X=\left(X_{t}, t \geq 0\right)$ is governed by the stochastic differential equation

$$
\mathrm{d} X_{t}=a X_{t} \mathrm{~d} t+b u_{t} \mathrm{~d} t+\mathrm{d} B_{t}^{H}, t \geq 0, X_{0}=x,
$$

which is as usual interpreted as an integral equation. Here $x$ is a fixed initial condition, $B^{H}=\left(B_{t}^{H}, t \geq 0\right)$ is a normalized fBm with the Hurst parameter $H$ in $[1 / 2,1)$ and the coefficients $a$ and $b \neq 0$ are fixed constants. We suppose that $X$ is completely observed and that a closed-loop control of the system is available in the sense

\footnotetext{
Keywords and phrases. Fractional Brownian motion, linear system, optimal control, quadratic payoff, infinite time.

1 Laboratoire de Statistique et Processus, Université du Maine, av. Olivier Messiaen, 72085 Le Mans Cedex 9, France; marina.kleptsyna@univ-lemans.fr

${ }^{2}$ Laboratoire de Modélisation et Calcul, Université J. Fourier, BP 53, 38041 Grenoble Cedex 9, France; Alain.Le-Breton@imag.fr

* She is also associated with the Institute of Information Transmission Problems, Moscow, Russia.

** A. Le Breton's research was supported by the Project IDOPT, CNRS-UJF-INPG-INRIA.
} 
that at each time $t \geq 0$ one may choose the input $u_{t}$ in view of the passed observations $\left\{X_{s}, 0 \leq s \leq t\right\}$ in order to drive the corresponding state, $X_{t}=X_{t}^{u}$ say. Then, given a cost function which evaluates the performance of the control actions, the classical problem of controlling the system dynamics so as to minimize this cost occurs. After considering in [10] the case of an expected integral quadratic cost on a finite time interval, here we analyze the case of an average quadratic payoff per unit time $J$ defined for a control policy $u=\left(u_{t}, t \geq 0\right)$ by

$$
J(u)=\limsup _{T \rightarrow+\infty} \frac{1}{T} \int_{0}^{T}\left[q X_{t}^{2}+r u_{t}^{2}\right] \mathrm{d} t,
$$

where $q$ and $r$ are positive constants. It is well-known that when $H=1 / 2$ and hence the noise in (1.1) is a Brownian motion, then (see, e.g., $[3,12]$ ) a solution $\bar{u}$ to the corresponding problem, called an optimal control, is provided for all $t \geq 0$ by the instantaneous linear feedback

$$
\bar{u}_{t}=-\frac{b}{r} \rho \bar{X}_{t} ; \quad \bar{X}_{t}=X_{t}^{\bar{u}}
$$

where $\rho$ is the nonnegative solution of the algebraic Riccati equation $\frac{b^{2}}{r} \rho^{2}-2 a \rho-q=0$, i.e.,

$$
\rho=\frac{r}{b^{2}}[a+\delta] ; \quad \delta=\sqrt{a^{2}+\frac{b^{2}}{r} q .}
$$

Moreover the optimal cost $J(\bar{u})$ is given by

$$
J(\bar{u})=\rho \quad \text { a.s. }
$$

Our main goal here is to show that actually when the system $(1.1)$ is driven by a fBm with some $H \in(1 / 2,1)$ instead of a Brownian motion, an explicit solution to the optimal control problem under the performance criterion (1.2) is still available.

The paper is organized as follows. At first in Section 2, we fix some notations and preliminaries. Then, in Section 3, a first solution to the concerned closed-loop control problem is elaborated: an optimal control is identified as a linear but not instantaneous feedback which involves the solution of a Volterra type integral equation and the optimal cost is computed. Section 4 is devoted to a complementary analysis: another optimal control defined in terms of a simpler and more explicite linear feedback is described and the lowest possible cost achievable by means of an instantaneous linear feedback is compared to the optimal cost. Finally, Section 5 is an Appendix dedicated to auxiliary developments: we derive some technical results and we investigate ergodic properties of some involved processes.

\section{Preliminaries}

In what follows all random variables and processes are defined on a given stochastic basis $(\Omega, \mathcal{F}, \mathbb{P})$. Moreover the natural filtration of a process is understood as the $\mathbb{P}$-completion of the filtration generated by this process.

Here, for some $H \in[1 / 2,1), B^{H}=\left(B_{t}^{H}, t \geq 0\right)$ is a normalized fractional Brownian motion with Hurst parameter $H$ means that $B^{H}$ is a Gaussian process with continuous paths such that $B_{0}^{H}=0, \mathbb{E} B_{t}^{H}=0$ and

$$
\mathbb{E} B_{s}^{H} B_{t}^{H}=\frac{1}{2}\left[s^{2 H}+t^{2 H}-|s-t|^{2 H}\right], s, t \geq 0 \text {. }
$$

Of course the fBm reduces to the standard Brownian motion when $H=1 / 2$. For $H \neq 1 / 2$, the fBm is outside the world of semimartingales but a theory of stochastic integration w.r. to fBm has been developed (see, e.g., [4] or [5]). Actually the case of deterministic integrands, which is sufficient for the purpose of the present paper, is easy to handle (see, e.g., [18]). 
- Fundamental martingale associated to $B^{H}$ - There are simple integral transformations which change the fBm to martingales (see $[9,16-19])$. In particular, defining for $0<s<t \leq T$

$$
\begin{gathered}
k_{H}(t, s)=\kappa_{H}^{-1} s^{\frac{1}{2}-H}(t-s)^{\frac{1}{2}-H} ; \kappa_{H}=2 H \Gamma(3 / 2-H) \Gamma(H+1 / 2), \\
w_{t}^{H}=\lambda_{H}^{-1} t^{2-2 H} ; \quad \lambda_{H}=\frac{2 H \Gamma(3-2 H) \Gamma(H+1 / 2)}{\Gamma(3 / 2-H)}, \\
M_{t}^{H}=\int_{0}^{t} k_{H}(t, s) \mathrm{d} B_{s}^{H},
\end{gathered}
$$

then the process $M^{H}$ is a Gaussian martingale, called in [18] the fundamental martingale, whose variance function $\left\langle M^{H}\right\rangle$ is nothing but the function $w^{H}$. Actually, the natural filtration of $M^{H}$ coincides with the natural filtration $\left(\mathcal{F}_{t}^{H}\right)$ of $B^{H}$. In particular, we have the direct consequence of the results of [9] that, given a suitably regular deterministic function $c=(c(t), t \geq 0)$, the following representation holds almost surely for $t \geq 0$ :

$$
\int_{0}^{t} c(s) \mathrm{d} B_{s}^{H}=\int_{0}^{t} K_{H}^{c}(t, s) \mathrm{d} M_{s}^{H}
$$

where for $H \in(1 / 2,1)$ the function $K_{H}^{c}$ is given by

$$
K_{H}^{c}(t, s)=H(2 H-1) \int_{s}^{t} c(r) r^{H-\frac{1}{2}}(r-s)^{H-\frac{3}{2}} \mathrm{~d} r, 0 \leq s \leq t
$$

and for $H=1 / 2$ the convention $K_{1 / 2}^{c}(t,.) \equiv c$ for all $t$ is used. Conversely, given a suitably regular deterministic function $f=(f(t), t \geq 0)$, we have also almost surely for $t \geq 0$ :

$$
\int_{0}^{t} f(s) \mathrm{d} M_{s}^{H}=\int_{0}^{t} k_{H}^{f}(t, s) \mathrm{d} B_{s}^{H}
$$

where the function $k_{H}^{f}$ is given by

$$
k_{H}^{f}(t, s)=-\kappa_{H}^{-1} s^{\frac{1}{2}-H} \frac{\mathrm{d}}{\mathrm{d} s} \int_{s}^{t}(r-s)^{\frac{1}{2}-H} f(r) \mathrm{d} r .
$$

- Admissible controls - Let $\mathcal{U}_{H}$ the class of $\left(\mathcal{F}_{t}^{H}\right)$-adapted processes $u=\left(u_{t}, t \geq 0\right)$ such that the stochastic differential equation (1.1) has a unique strong solution $X^{u}$. Of course then $X^{u}$ is a $\left(\mathcal{F}_{t}^{H}\right)$-adapted process. Actually, as mentioned in Section 1, for control purpose we are interested in closed-loop policies. So, we introduce the class of admissible controls as the class $\mathcal{U}_{a d}$ of those $u$ 's in $\mathcal{U}_{H}$ which are $\left(\mathcal{F}_{t}^{u}\right)$-adapted processes where $\left(\mathcal{F}_{t}^{u}\right)$ is the natural filtration of the corresponding state process $X^{u}$. For $u \in \mathcal{U}_{a d}$, the pair $\left(u, X^{u}\right)$ is called an admissible pair and if $\bar{u} \in \mathcal{U}_{a d}$ is such that

$$
J(\bar{u})=\inf \left\{J(u), u \in \mathcal{U}_{a d}\right\} \quad \text { a.s. }
$$

then it is called an optimal control and $(\bar{u}, \bar{X})$, where $\bar{X}=X^{\bar{u}}$, is called an optimal pair and the quantity $J(\bar{u})$ is called the optimal cost.

\section{First solution of the optimal Control Problem}

To define a control policy as a candidate for optimality in the stated infinite time horizon problem, starting from the solution of the finite horizon time problem which is derived in [10], one may take benefit of an heuristics based on the connection between these two problems in the standard case $H=1 / 2$. Then it appears natural 
to introduce here the following families $(\gamma(., s), s \geq 0)$ and $(\bar{k}(., s), s \geq 0)$ of auxiliary deterministic functions. For any fixed $s \geq 0$, the function $\gamma(., s)=(\gamma(t, s), t \geq s)$ is defined by

$$
\gamma(t, s)=\delta \mathrm{e}^{\delta t} \int_{t}^{\infty} \mathrm{e}^{-\delta \tau} K_{H}(\tau, s) \mathrm{d} \tau,
$$

where $\delta$ is given by (1.4) and the function $K_{H}$ is given by (2.6) for $c \equiv 1$, i.e.,

$$
K_{H}(\tau, s)=H(2 H-1) \int_{s}^{\tau} r^{H-\frac{1}{2}}(r-s)^{H-\frac{3}{2}} \mathrm{~d} r, 0 \leq s \leq \tau .
$$

Now, for any fixed $s \geq 0$, the function $\bar{k}(., s)=(\bar{k}(t, s), t \geq s)$ is obtained by substituting $\gamma(r, r)$ for $f(r)$ in the definition (2.8), i.e.,

$$
\bar{k}(t, s)=-\kappa_{H}^{-1} s^{\frac{1}{2}-H} \frac{\mathrm{d}}{\mathrm{d} s} \int_{s}^{t}(r-s)^{\frac{1}{2}-H} \gamma(r, r) \mathrm{d} r .
$$

Observe that, due to $(2.5)$ and $(2.7)$, the functions $K_{H}(t,$.$) and \bar{k}(t,$.$) allow the representations almost surely$ for all $t \geq 0$

$$
B_{t}^{H}=\int_{0}^{t} K_{H}(t, s) \mathrm{d} M_{s}^{H} ; \quad \int_{0}^{t} \gamma(s, s) \mathrm{d} M_{s}^{H}=\int_{0}^{t} \bar{k}(t, s) \mathrm{d} B_{s}^{H} .
$$

Moreover, it can be checked that the following property also holds almost surely

$$
\int_{0}^{+\infty} \mathrm{e}^{-\delta s} \gamma(s, s) \mathrm{d} M_{s}^{H}=\int_{0}^{+\infty} \mathrm{e}^{-\delta s} \mathrm{~d} B_{s}^{H} .
$$

Now, we may state our main result:

Theorem 3.1. Let the pair $(\bar{u}, \bar{X})$ be governed by the system

$$
\begin{gathered}
\bar{u}_{t}=-\frac{b}{r} \rho\left[\bar{X}_{t}+\bar{v}_{t}\right] ; \quad \bar{X}_{t}=X_{t}^{\bar{u}}, \\
\bar{v}_{t}=\int_{0}^{t} \delta \bar{v}_{s} \mathrm{~d} s+\int_{0}^{t}[\bar{k}(t, s)-1]\left\{\mathrm{d} \bar{X}_{s}-\left[a \bar{X}_{s}+b \bar{u}_{s}\right] \mathrm{d} s\right\},
\end{gathered}
$$

where $(\rho, \delta)$ and $\bar{k}$ are defined by (1.4) and (3.3) respectively. Then the control $\bar{u}$ is optimal in $\mathcal{U}_{\text {ad }}$ and $(\bar{u}, \bar{X})$ is an optimal pair. Moreover the optimal cost is given by

$$
J(\bar{u})=\bar{\lambda} \quad \text { a.s. },
$$

where $\bar{\lambda}$ is the constant

$$
\bar{\lambda}=\frac{q \Gamma(2 H+1)}{2 \delta^{2 H}}\left[1+\frac{\delta+a}{\delta-a} \sin \pi H\right] .
$$

Remark 3.1. (a) Observe that in the case $H=1 / 2$, for all $0 \leq s \leq t$, the entries $\gamma(t, s)$ and $\bar{k}(t, s)$ both reduce to 1 . Hence, it is readily seen that $\bar{v} \equiv 0, \bar{u}=-(b / r) \rho \bar{X}$ and also $\bar{\lambda}=\rho$. So, finally, the statement in Theorem 3.1 reduces to the well-known result recalled in Section 1.

(b) It is worth mentioning that actually the additional term $\bar{v}_{t}$ which appears in the case $H>1 / 2$ can be interpreted in terms of the predictors at time $t$ of the noise component $B_{\tau}^{H}, \tau \geq t$ based on the observed optimal dynamics $\left(\bar{X}_{s}, s \leq t\right)$ up to time $t$. Precisely, one can rewrite

$$
\bar{v}_{t}=\int_{t}^{+\infty} \mathrm{e}^{-\delta(\tau-t)} \mathrm{d} \hat{B}_{\tau}^{t} ; \quad \hat{B}_{\tau}^{t}=\mathbb{E}\left(B_{\tau}^{H} / \mathcal{F}_{t}^{\bar{u}}\right), \tau \geq t .
$$


or equivalently

$$
\bar{v}_{t}=\mathbb{E}\left(\xi_{t} / \mathcal{F}_{t}^{\bar{u}}\right)
$$

where

$$
\xi_{t}=\int_{t}^{+\infty} \mathrm{e}^{-\delta(\tau-t)} \mathrm{d} B_{\tau}^{H} .
$$

This will be made clear in Remark 3.2 after the proof of Lemma 3.1.

The proof of Theorem 3.1 is organized through several lemmas. At first, given some process $u \in \mathcal{U}_{H}$ and the corresponding $X^{u}$, we introduce the $\left(\mathcal{F}_{t}^{H}\right)$-adapted process $p=\left(p_{t}, t \geq 0\right)$ as the solution of the stochastic differential equation:

$$
\mathrm{d} p_{t}=-a p_{t} \mathrm{~d} t-q X_{t}^{u} \mathrm{~d} t+\rho \gamma(t, t) \mathrm{d} M_{t}^{H}, t \geq 0 ; p_{0}=\rho x .
$$

Lemma 3.1. There exists a unique process $\bar{u} \in \mathcal{U}_{H}$ such that $\bar{u}$ can be represented as $\bar{u}=-(b / r) \bar{p}$ where $\bar{p}$ is the process satisfying the equation (3.13) which corresponds to $\bar{X}=X^{\bar{u}}$. Moreover, the pair $(\bar{u}, \bar{X})$ is admissible and is governed by the system (3.6)-(3.7).

Proof. At first, we prove the uniqueness. Suppose that $u^{1}$ and $u^{2}$ in $\mathcal{U}_{H}$ both satisfy the property which is required in the first assertion, i.e., for $i=1,2$, we have $u^{i}=-(b / r) p^{i}$, where $p^{i}$ satisfies equation (3.13) with $X^{i}=X^{u^{i}}$ in place of $X^{u}$. Let us use the notations

$$
\Delta X=X^{1}-X^{2} ; \Delta p=p^{1}-p^{2}
$$

From equations (1.1) and (3.13), we have

$$
\begin{aligned}
\mathrm{d} \Delta X_{t} & =a \Delta X_{t} \mathrm{~d} t-\frac{b^{2}}{r} \Delta p_{t} \mathrm{~d} t, t \geq 0 ; \Delta X_{0}=0, \\
\mathrm{~d} \Delta p_{t} & =-a \Delta p_{t} \mathrm{~d} t-q \Delta X_{t} \mathrm{~d} t, t \geq 0, \Delta p_{0}=0 .
\end{aligned}
$$

Of course, consequently we have $\Delta X_{t} \equiv 0$ and $\Delta p_{t} \equiv 0$, which means in particular that $u^{1} \equiv u^{2}$.

Now we turn to prove the existence. We take

$$
\bar{u}_{t}=-\frac{b}{r} \bar{p}_{t} ; \quad \bar{p}_{t}=\rho\left(\tilde{X}_{t}+V_{t}\right) ; \quad V_{t}=\int_{0}^{t} \gamma(t, s) \mathrm{d} M_{s}^{H}
$$

where

$$
\mathrm{d} \widetilde{X}_{t}=a\left[\widetilde{X}_{t}+B_{t}^{H}\right] \mathrm{d} t-\frac{b^{2}}{r} \rho\left[\widetilde{X}_{t}+V_{t}\right] \mathrm{d} t ; \widetilde{X}_{0}=x .
$$

Observe that actually, from (3.15)-(3.16), it appears that the process $\bar{X}=\widetilde{X}+B^{H}$ is nothing but the state process $\bar{X}=X^{\bar{u}}$ which corresponds to $\bar{u}$ by equation (1.1). Moreover, due to the representation (3.4) of $B^{H}$, it is readily seen that we can rewrite $\bar{p}$ in terms of $\bar{X}$ in the form

$$
\bar{p}_{t}=\rho \bar{X}_{t}+\int_{0}^{t}\left[\gamma(t, s)-\rho K_{H}(t, s)\right] \mathrm{d} M_{s}^{H} .
$$

Now, we show that the process $\bar{p}$ is a semimartingale and we identify its decomposition. From (3.1), it is easy to see that $\gamma(., s)$ is differentiable with

$$
\dot{\gamma}(t, s)=\delta\left[\gamma(t, s)-K_{H}(t, s)\right]
$$


Hence, $V$ defined in (3.15) is a semimartingale and its stochastic differential is

$$
\begin{aligned}
\mathrm{d} V_{t} & =\left[\int_{0}^{t} \dot{\gamma}(t, s) \mathrm{d} M_{s}^{H}\right] \mathrm{d} t+\gamma(t, t) \mathrm{d} M_{t}^{H} \\
& =\left\{\int_{0}^{t} \delta\left[\gamma(t, s)-K_{H}(t, s)\right] \mathrm{d} M_{s}^{H}\right\} \mathrm{d} t+\gamma(t, t) \mathrm{d} M_{t}^{H} .
\end{aligned}
$$

Then, again using the definition (3.15) of $V$ and taking into account the representation (3.4) of $B^{H}$, we get

$$
\mathrm{d} V_{t}=\delta\left[V_{t}-B_{t}^{H}\right] \mathrm{d} t+\gamma(t, t) \mathrm{d} M_{t}^{H} .
$$

Now, from the definition (3.15) of $\bar{p}$, we have

$$
\mathrm{d} \bar{p}_{t}=\rho\left[\mathrm{d} \widetilde{X}_{t}+\mathrm{d} V_{t}\right]
$$

and, inserting (3.16) and (3.18), we can compute this stochastic differential. Using the definition (1.4) of $(\rho, \delta)$, it is easy to check that actually

$$
\mathrm{d} \bar{p}_{t}=-a \bar{p}_{t} \mathrm{~d} t-q\left[\widetilde{X}_{t}+B_{t}^{H}\right] \mathrm{d} t+\rho \gamma(t, t) \mathrm{d} M_{t}^{H} .
$$

This means exactly that $\mathrm{d} \bar{p}_{t}$ is given by the right-hand side of equation (3.13) with $\bar{X}_{t}=\widetilde{X}_{t}+B_{t}^{H}$. Summarizing, we have checked that the $\left(\mathcal{F}_{t}^{H}\right)$-adapted process $\bar{p}$ defined in (3.17) satisfies equation (3.13) with the state process $\bar{X}$ corresponding to $\bar{u}=-(b / r) \bar{p}$ in place of $X^{u}$.

Now we turn to show the second assertion in the statement. To do this, it suffices to prove that $\bar{u}$ is actually the closed-loop control defined in (3.6)-(3.7) since then it turns that $\bar{u} \in \mathcal{U}_{a d}$ and hence the pair $(\bar{u}, \bar{X})$ is admissible. From (3.17), we rewrite $\bar{p}_{t}=\rho\left(\bar{X}_{t}+\bar{v}_{t}\right)$ where

$$
\bar{v}_{t}=V_{t}-B_{t}^{H} .
$$

From the stochastic differential (3.18) for $V$, computing $\mathrm{d} \bar{v}_{t}=\mathrm{d} V_{t}-\mathrm{d} B_{t}^{H}$, it is readily seen that

$$
\bar{v}_{t}=\int_{0}^{t} \delta \bar{v}_{s} \mathrm{~d} s+\int_{0}^{t} \gamma(s, s) \mathrm{d} M_{s}^{H}-B_{t}^{H} .
$$

But, due to the representation (3.4) of the stochastic integral with respect to $M^{H}$ as an integral with respect to $B^{H}$, in terms of the function $\bar{k}$ defined by (3.3), we can rewrite this as

$$
\bar{v}_{t}=\int_{0}^{t} \delta \bar{v}_{s} \mathrm{~d} s+\int_{0}^{t}[\bar{k}(t, s)-1] \mathrm{d} B_{s}^{H} .
$$

Consequently, since due to (1.1) we have $\mathrm{d} B_{s}^{H}=\mathrm{d} \bar{X}_{s}-\left[a \bar{X}_{s}+b \bar{u}_{s}\right] \mathrm{d} s$, it means that the pair $(\bar{u}, \bar{X})$ is governed by the system (3.6)-(3.7) and in particular this pair is admissible.

Remark 3.2. Let us justify the observation which is formulated in Remark 3.1. Actually, the component $\bar{v}_{t}$ defined by (3.19) in the previous proof is nothing but

$$
\bar{v}_{t}=\int_{0}^{t}\left[\gamma(t, s)-K_{H}(t, s)\right] \mathrm{d} M_{s}^{H} .
$$


From (3.1), it is easy to see that

$$
\gamma(t, s)-K_{H}(t, s)=\mathrm{e}^{\delta t} \int_{t}^{+\infty} \mathrm{e}^{-\delta \tau} \dot{K}_{H}(\tau, s) \mathrm{d} \tau, s \leq t
$$

where the function $\dot{K}_{H}(\tau, s)$ is the derivative of $K_{H}(\tau, s)$ with respect to $\tau$, i.e.,

$$
\dot{K}_{H}(\tau, s)=H(2 H-1) \tau^{H-\frac{1}{2}}(\tau-s)^{H-\frac{3}{2}}, 0 \leq s \leq \tau .
$$

Consequently, we can write

or

$$
\bar{v}_{t}=\int_{0}^{t}\left\{\int_{t}^{+\infty} \mathrm{e}^{-\delta(\tau-t)} \dot{K}_{H}(\tau, s) \mathrm{d} \tau\right\} \mathrm{d} M_{s}^{H}
$$

$$
\bar{v}_{t}=\int_{t}^{+\infty} \mathrm{e}^{-\delta(\tau-t)}\left\{\int_{0}^{t} \dot{K}_{H}(\tau, s) \mathrm{d} M_{s}^{H}\right\} \mathrm{d} \tau .
$$

But, due to the representation $(2.5)$ of $B$, it is clear that for every $\tau \geq t$, the predictor $\mathbb{E}\left(B_{\tau} / \mathcal{F}_{t}^{H}\right)$ of $B_{\tau}$ based on the observation of $B^{H}$ on $[0, t]$ is given by

$$
\mathbb{E}\left(B_{\tau} / \mathcal{F}_{t}^{H}\right)=\int_{0}^{t} K_{H}(\tau, s) \mathrm{d} M_{s}^{H} ; \quad \frac{\mathrm{d} \mathbb{E}\left(B_{\tau} / \mathcal{F}_{t}^{H}\right)}{\mathrm{d} \tau}=\int_{0}^{t} \dot{K}_{H}(\tau, s) \mathrm{d} M_{s}^{H}
$$

and so

$$
\bar{v}_{t}=\int_{t}^{+\infty} \mathrm{e}^{-\delta(\tau-t)} \mathrm{d} \mathbb{E}\left(B_{\tau} / \mathcal{F}_{t}^{H}\right) .
$$

Finally, since by construction the solution $\bar{X}=X^{\bar{u}}$ of (1.1) for $u=\bar{u}$ is such that $\mathcal{F}_{t}^{\bar{u}}=\mathcal{F}_{t}^{H}$, we can represent $\bar{v}_{t}$ in the form (3.10) which was claimed in Remark 3.1.

Now we analyze the asymptotic behavior of the admissible pair $(\bar{u}, \bar{X})$ in order to show that it achieves the announced lower bound $\bar{\lambda}$ for the cost.

Lemma 3.2. Let $(\bar{u}, \bar{X})$ be the admissible pair governed by (3.6)-(3.7). Then, the following property holds:

$$
\lim _{T \rightarrow+\infty} \frac{1}{T} \int_{0}^{T}\left[q \bar{X}_{t}^{2}+r \bar{u}_{t}^{2}\right] \mathrm{d} t=\bar{\lambda} \quad \text { a.s. },
$$

where $\bar{\lambda}$ is given by (3.9) and consequently, for $J$ evaluated according to (1.2), the equality (3.8) holds.

Proof. At first we derive a convenient representation of the pair $(\bar{u}, \bar{X})$. From the system (3.6)-(3.7), it is readily seen that

and so

$$
\mathrm{d} \bar{X}_{t}=-\delta \bar{X}_{t} \mathrm{~d} t-\frac{b^{2}}{r} \rho \bar{v}_{t} \mathrm{~d} t+\mathrm{d} B_{t}^{H}
$$

$$
\bar{X}_{t}=\mathrm{e}^{-\delta t}\left\{x-\frac{b^{2}}{r} \rho \int_{0}^{t} \mathrm{e}^{\delta s} \bar{v}_{s} \mathrm{~d} s+\int_{0}^{t} \mathrm{e}^{\delta s} \mathrm{~d} B_{s}^{H}\right\} .
$$

From (3.20), integrating by parts, it is easy to check that

$$
\int_{0}^{t} \mathrm{e}^{\delta s} \bar{v}_{s} \mathrm{~d} s=\frac{1}{2 \delta}\left\{\mathrm{e}^{\delta t} \bar{v}_{t}-\int_{0}^{t} \mathrm{e}^{\delta s} \rho\left[\gamma(s, s) \mathrm{d} M_{s}^{H}-\mathrm{d} B_{s}^{H}\right]\right\},
$$

and hence to get the representation

$$
\bar{X}_{t}=-\frac{\delta+a}{2 \delta} \bar{v}_{t}+\frac{\delta+a}{2 \delta} \bar{w}_{t}+\frac{\delta-a}{2 \delta} \bar{z}_{t},
$$


where

$$
\bar{w}_{t}=\mathrm{e}^{-\delta t}\left\{x+\int_{0}^{t} \mathrm{e}^{\delta s} \gamma(s, s) \mathrm{d} M_{s}^{H}\right\} ; \quad \bar{z}_{t}=\mathrm{e}^{-\delta t}\left\{x+\int_{0}^{t} \mathrm{e}^{\delta s} \mathrm{~d} B_{s}^{H}\right\} .
$$

Moreover, since $\bar{u}=-(b / r) \rho(\bar{X}+\bar{v})$, we have also

$$
\bar{u}_{t}=-\frac{b}{r} \rho\left\{\frac{\delta-a}{2 \delta} \bar{v}_{t}+\frac{\delta+a}{2 \delta} \bar{w}_{t}+\frac{\delta-a}{2 \delta} \bar{z}_{t}\right\}
$$

It follows from (3.23) and (3.25) that

$$
b \bar{u}_{t}+(\delta+a) \bar{w}_{t}=(\delta-a)\left(\bar{X}_{t}-\bar{z}_{t}\right)
$$

and

From (3.26)-(3.27), we get that

$$
\frac{\mathrm{d}}{\mathrm{d} t}\left(\bar{X}_{t}-\bar{z}_{t}\right)=\delta\left(\bar{X}_{t}-\bar{z}_{t}\right)+(\delta+a)\left(\bar{z}_{t}-\bar{w}_{t}\right), \quad \bar{X}_{0}-\bar{z}_{0}=0 .
$$

$$
q \bar{X}_{t}^{2}+r \bar{u}_{t}^{2}=q \bar{X}_{t}^{2}+\frac{r}{b^{2}}\left[(\delta-a)\left(\bar{X}_{t}-\bar{z}_{t}\right)-(\delta+a) \bar{w}_{t}\right]^{2},
$$

and

$$
\frac{\mathrm{d}}{\mathrm{d} t}\left(\bar{X}_{t}-\bar{z}_{t}\right)^{2}=2\left(\bar{X}_{t}-\bar{z}_{t}\right)\left[\delta\left(\bar{X}_{t}-\bar{z}_{t}\right)+(\delta+a)\left(\bar{z}_{t}-\bar{w}_{t}\right)\right], \quad\left(\bar{X}_{0}-\bar{z}_{0}\right)^{2}=0 .
$$

Thus, by difference, we obtain

$$
q \bar{X}_{t}^{2}+r \bar{u}_{t}^{2}-\frac{q}{\delta+a} \frac{\mathrm{d}}{\mathrm{d} t}\left(\bar{X}_{t}-\bar{z}_{t}\right)^{2}=q\left[\bar{z}_{t}^{2}+\frac{\delta+a}{\delta-a} \bar{w}_{t}^{2}\right],
$$

which by integration gives that

$$
\int_{0}^{T}\left[q \bar{X}_{t}^{2}+r \bar{u}_{t}^{2}\right] \mathrm{d} t=q \int_{0}^{T}\left[\bar{z}_{t}^{2}+\frac{\delta+a}{\delta-a} \bar{w}_{t}^{2}\right] \mathrm{d} t+\frac{q}{\delta+a}\left(\bar{X}_{T}-\bar{z}_{T}\right)^{2} .
$$

So, to prove the statement, we determine successively the limits

$$
\lim _{T \rightarrow+\infty} \frac{1}{T} \int_{0}^{T} \bar{z}_{t}^{2} \mathrm{~d} t, \quad \lim _{T \rightarrow+\infty} \frac{1}{T} \int_{0}^{T} \bar{w}_{t}^{2} \mathrm{~d} t, \quad \lim _{T \rightarrow+\infty} \frac{1}{T}\left(\bar{X}_{T}-\bar{z}_{T}\right)^{2}
$$

Choosing a process $\left(B_{t}^{H}, t \leq 0\right)$ such that $\left(B_{t}^{H}, t \in \mathbb{R}\right)$ is a two-sided fractional Brownian motion, we can define a Gaussian stationary ergodic process $\left(z_{t}^{*}, t \in \mathbb{R}\right)$ by

$$
z_{t}^{*}=\int_{-\infty}^{t} \mathrm{e}^{-\delta(t-s)} \mathrm{d} B_{s}^{H} .
$$

Thanks to Proposition 5.2 of the Appendix and to the Birkhoff theorem, the following properties hold:

$$
\lim _{T \rightarrow+\infty} \frac{z_{T}^{*}}{\sqrt{T}}=0 \quad \text { a.s. }
$$

and

$$
\lim _{T \rightarrow+\infty} \frac{1}{T} \int_{0}^{T}\left|z_{t}^{*}\right|^{2} \mathrm{~d} t=\mathbb{E}\left\{\left|z_{0}^{*}\right|^{2}\right\} \quad \text { a.s. }
$$


where $\mathbb{E}\left\{\left|z_{0}^{*}\right|^{2}\right\}$ can be computed as

$$
\mathbb{E}\left\{\left|z_{0}^{*}\right|^{2}\right\}=H(2 H-1) \int_{0}^{\infty} \int_{0}^{\infty} \mathrm{e}^{-\delta(s+r)}|s-r|^{2 H-2} \mathrm{~d} r \mathrm{~d} s=\frac{\Gamma(2 H+1)}{2 \delta^{2 H}}
$$

From (3.24) we see that

$$
\lim _{t \rightarrow+\infty}\left(\bar{z}_{t}-z_{t}^{*}\right)=\lim _{t \rightarrow+\infty} \mathrm{e}^{-\delta t}\left\{x-\int_{-\infty}^{0} \mathrm{e}^{\delta s} \mathrm{~d} B_{s}^{H}\right\}=0 \quad \text { a.s. }
$$

and thus we have also

$$
\lim _{T \rightarrow+\infty} \frac{\bar{z}_{T}}{\sqrt{T}}=0 \quad \text { a.s. }
$$

and

$$
\lim _{T \rightarrow+\infty} \frac{1}{T} \int_{0}^{T} \bar{z}_{t}^{2} \mathrm{~d} t=\frac{\Gamma(2 H+1)}{2 \delta^{2 H}} \quad \text { a.s. }
$$

Assertion (iii) in Proposition 5.1 of the Appendix says that

$$
\lim _{T \rightarrow+\infty} \frac{1}{T} \int_{0}^{T} \bar{w}_{t}^{2} \mathrm{~d} t=\frac{\Gamma(2 H+1)}{2 \delta^{2 H}} \sin \pi H \quad \text { a.s. }
$$

Now, we show that

$$
\lim _{T \rightarrow+\infty} \frac{\bar{X}_{T}-\bar{z}_{T}}{\sqrt{T}}=0 \quad \text { a.s. }
$$

From equation (3.27), we have

$$
\bar{X}_{t}-\bar{z}_{t}=(\delta+a) \mathrm{e}^{\delta t} \int_{0}^{t} \mathrm{e}^{-\delta s}\left[\bar{z}_{s}-\bar{w}_{s}\right] \mathrm{d} s .
$$

But, from (3.5) and (3.24), one can see that

$$
\int_{0}^{+\infty} \mathrm{e}^{-\delta s}\left[\bar{z}_{s}-\bar{w}_{s}\right] \mathrm{d} s=0
$$

and so the following representation holds:

$$
\bar{X}_{t}-\bar{z}_{t}=-(\delta+a) \mathrm{e}^{\delta t} \int_{t}^{+\infty} \mathrm{e}^{-\delta s}\left[\bar{z}_{s}-\bar{w}_{s}\right] \mathrm{d} s .
$$

Assertion (ii) in Proposition 5.1 of the Appendix says that

$$
\lim _{T \rightarrow+\infty} \frac{\bar{w}_{T}}{\sqrt{T}}=0 \quad \text { a.s. }
$$

Hence, due to (3.30), we have also that

$$
\lim _{T \rightarrow+\infty} \frac{\bar{z}_{T}-\bar{w}_{T}}{\sqrt{T}}=0 \quad \text { a.s. }
$$


Now, (3.33) is a direct consequence of (3.35) and (3.37). Finally, inserting relations (3.31), (3.32) and (3.33) into (3.28), it turns that

$$
\lim _{T \rightarrow+\infty} \frac{1}{T} \int_{0}^{T}\left[q \bar{X}_{t}^{2}+r \bar{u}_{t}^{2}\right] \mathrm{d} t=\frac{q \Gamma(2 H+1)}{2 \delta^{2 H}}\left[1+\frac{\delta+a}{\delta-a} \sin \pi H\right] \quad \text { a.s. },
$$

where the right hand side is nothing but the constant $\bar{\lambda}$ given by (3.9).

Finally, to finish the proof of Theorem 3.1, it remains to show that the process $\bar{u}$ involved in the above statements minimizes $J$ over $\mathcal{U}_{a d}$.

Lemma 3.3. Let $(\bar{u}, \bar{X})$ be the admissible pair governed by (3.6)-(3.7). Then $\bar{u}$ minimizes $J$ over $\mathcal{U}_{H}$ and therefore $(\bar{u}, \bar{X})$ is an optimal pair.

Proof. Given an arbitrary $u \in \mathcal{U}_{H}$, we use the notation

$$
J_{T}(u)=\int_{0}^{T}\left[q X_{t}^{2}+r u_{t}^{2}\right] \mathrm{d} t
$$

where $X_{t}=X_{t}^{u}$. We evaluate the difference

$$
J_{T}(u)-J_{T}(\bar{u})=\int_{0}^{T}\left\{q\left[X_{t}^{2}-\bar{X}_{t}^{2}\right]+r\left[u_{t}^{2}-\bar{u}_{t}^{2}\right]\right\} \mathrm{d} t .
$$

Using the equality $y^{2}-\bar{y}^{2}=(y-\bar{y})^{2}+2 \bar{y}(y-\bar{y})$ and exploiting the property $\bar{u}=-(b / r) \bar{p}$, it is readily seen that

where

$$
J_{T}(u)-J_{T}(\bar{u})=\Delta_{1}(T)+2 \Delta_{2}(T)
$$

$$
\begin{aligned}
& \Delta_{1}(T)=\int_{0}^{T}\left\{q\left[X_{t}-\bar{X}_{t}\right]^{2}+r\left[u_{t}-\bar{u}_{t}\right]^{2}\right\} \mathrm{d} t, \\
& \Delta_{2}(T)=\int_{0}^{T}\left\{q \bar{X}_{t}\left[X_{t}-\bar{X}_{t}\right]-b \bar{p}_{t}\left[u_{t}-\bar{u}_{t}\right]\right\} \mathrm{d} t .
\end{aligned}
$$

Since $J(u)=\lim \sup _{T \rightarrow+\infty} T^{-1} J_{T}(u)$ a.s. and $\Delta_{1}(T) \geq 0$, of course we have

$$
J(u) \geq J(\bar{u})+\liminf _{T \rightarrow+\infty} T^{-1} \Delta_{2}(T) \quad \text { a.s. }
$$

Hence, to prove that $\bar{u}$ minimizes $J$ over $\mathcal{U}_{H}$, it is sufficient to show that $\lim _{T \rightarrow+\infty} T^{-1} \Delta_{2}(T)=0$ a.s. But, rewriting the quantity in the last integral above as

$$
\left(X_{t}-\bar{X}_{t}\right)\left[q \bar{X}_{t}+a \bar{p}_{t}\right]-\bar{p}_{t}\left[a\left(X_{t}-\bar{X}_{t}\right)+b\left(u_{t}-\bar{u}_{t}\right)\right],
$$

and taking into account equations (1.1) and (3.13), we see that $\Delta_{2}(T)$ can be written as

$$
\Delta_{2}(T)=-\int_{0}^{T}\left(X_{t}-\bar{X}_{t}\right) \mathrm{d} \bar{p}_{t}-\int_{0}^{T} \bar{p}_{t} \mathrm{~d}\left(X_{t}-\bar{X}_{t}\right)+\rho \int_{0}^{T}\left(X_{t}-\bar{X}_{t}\right) \gamma(t, t) \mathrm{d} M_{t}^{H} .
$$

Now, integrating by parts, since $X_{0}-\bar{X}_{0}=0$, it comes that

$$
\Delta_{2}(T)=-\bar{p}_{T}\left(X_{T}-\bar{X}_{T}\right)+\rho \int_{0}^{T}\left(X_{t}-\bar{X}_{t}\right) \gamma(t, t) \mathrm{d} M_{t}^{H} .
$$


Hence of course it suffices to show that if the admissible pair $(u, X)$ is such that $J(u)<+\infty$ a.s., then

$$
\lim _{T \rightarrow+\infty} T^{-1} \bar{p}_{T}\left(X_{T}-\bar{X}_{T}\right)=0 \quad \text { a.s. }
$$

and

$$
\lim _{T \rightarrow+\infty} T^{-1} \int_{0}^{T}\left(X_{t}-\bar{X}_{t}\right) \gamma(t, t) \mathrm{d} M_{t}^{H}=0 \quad \text { a.s. }
$$

In order to prove (3.38), at first let us note that for such a pair $(u, X)$ we have

$$
\limsup _{T \rightarrow+\infty} \frac{1}{T} \int_{0}^{T}\left[q\left(X_{t}-\bar{X}_{t}\right)^{2}+r\left(u_{t}-\bar{u}_{t}\right)^{2}\right] \mathrm{d} t<+\infty \quad \text { a.s. }
$$

Therefore, defining $\zeta_{t}=X_{t}-\bar{X}_{t}$ and, due to (1.1), rewriting $b\left(u_{t}-\bar{u}_{t}\right)$ as $\dot{\zeta}_{t}-a \zeta_{t}$, we get

$$
\limsup _{T \rightarrow+\infty} \frac{1}{T} \int_{0}^{T} \frac{r}{b^{2}}\left[\left(\dot{\zeta}_{t}-\delta \zeta_{t}\right)^{2}+2(\delta-a) \zeta_{t} \dot{\zeta}_{t}\right] \mathrm{d} t<+\infty \quad \text { a.s. }
$$

Thus

$$
\limsup _{T \rightarrow+\infty} \frac{1}{T} \int_{0}^{T}\left(\dot{\zeta}_{t}-\delta \zeta_{t}\right)^{2} \mathrm{~d} t+\limsup _{T \rightarrow+\infty} \frac{\zeta_{T}^{2}}{T}<+\infty \quad \text { a.s. }
$$

and in particular $\lim \sup _{T \rightarrow+\infty} T^{-1} \zeta_{T}^{2}<+\infty \quad$ a.s., which means

$$
\limsup _{T \rightarrow+\infty} \frac{X_{T}-\bar{X}_{T}}{\sqrt{T}}<+\infty \text { a.s. }
$$

Hence to get (3.38) it is sufficient to show that

$$
\lim _{T \rightarrow+\infty} \frac{\bar{p}_{T}}{\sqrt{T}}=0 \quad \text { a.s. }
$$

But, since $\bar{p}_{t}=-(r / b) \bar{u}_{t}$, it is an immediate consequence of (3.26), (3.33) and (3.36).

In order to prove (3.39), we rewrite it as

$$
\lim _{T \rightarrow+\infty} \frac{N_{T}}{\langle N\rangle_{T}} \frac{\langle N\rangle_{T}}{T}=0 \quad \text { a.s. }
$$

where $\left(N_{t}, t \geq 0\right)$ is the martingale defined by

$$
N_{t}=\int_{0}^{t}\left(\bar{X}_{s}-X_{s}\right) \gamma(s, s) \mathrm{d} M_{s}^{H}
$$

with the quadratic variation process $\left(\langle N\rangle_{t}, t \geq 0\right)$ given by

$$
\langle N\rangle_{t}=\int_{0}^{t}\left(\bar{X}_{s}-X_{s}\right)^{2} \gamma^{2}(s, s) \mathrm{d}\left\langle M^{H}\right\rangle_{s} .
$$

Due to assertion $(i)$ in Proposition 5.1 of the Appendix, we have

$$
\limsup _{T \rightarrow+\infty} \frac{\langle N\rangle_{T}}{T}<+\infty \quad \text { a.s. on }\left\{\langle N\rangle_{T} \rightarrow+\infty\right\}
$$

and so (3.39) follows immediately from Lemma 2.6.3 in [14]. 
Remark 3.3. (a) One may observe that actually the optimal pair $(\bar{u}, \bar{X})$ is an asymptotically stationarity process in the sense that, for $t-s$ fixed, as $s$ tends to $+\infty$, all the covariances converge to a limit.

(b) It can also be checked that the pair $(\bar{u}, \bar{X})$ is also optimal with respect to the averaged quadratic criterion

$$
\limsup _{T \rightarrow+\infty} \frac{1}{T} \mathbb{E}\left\{\int_{0}^{T}\left[q X_{t}^{2}+r u_{t}^{2}\right] \mathrm{d} t\right\} .
$$

Moreover, the corresponding minimum value of the cost is again the constant $\bar{\lambda}$ given by (3.9).

\section{Second Solution of the optimal Control Problem}

The analysis in the previous section exhibits a solution of the problem such that the optimal pair $(\bar{u}, \bar{X})$ is a Gaussian asymptotically stationary process (see Rem. 3.3(a)). So it seems rather natural to look for another solution of the problem in the class of those $u$ 's in $\mathcal{U}_{a d}$ for which the pair $\left(u, X^{u}\right)$ is clearly candidate to have this property. Moreover, it appears (see, e.g., representations (3.23) and (3.25)) that both components of the pair $(\bar{u}, \bar{X})$ can be written in terms of integrals with respect to $B^{H}$. Globally, these observations lead to attempt to find an optimal pair in the class of processes $(u, X)$ which can be represented as ${ }^{1}$

$$
u_{t}=\int_{0}^{t} \mathcal{U}(t-s) \mathrm{d} B_{s}^{H} ; \quad X_{t}=\int_{0}^{t} \mathcal{X}(t-s) \mathrm{d} B_{s}^{H},
$$

where $\mathcal{U}$ and $\mathcal{X}$ are appropriate deterministic functions. Of course, we are interested in pairs $(\mathcal{U}, \mathcal{X})$ for which, choosing a process $\left(B_{t}^{H}, t \leq 0\right)$ such that $\left(B_{t}^{H}, t \in \mathbb{R}\right)$ is a two-sided fractional Brownian motion, the process $(\hat{u}, \hat{X})$ where

$$
\hat{u}_{t}=\int_{-\infty}^{t} \mathcal{U}(t-s) \mathrm{d} B_{s}^{H} ; \quad \hat{X}_{t}=\int_{-\infty}^{t} \mathcal{X}(t-s) \mathrm{d} B_{s}^{H},
$$

is an ergodic stationary process with the same asymptotic behaviour as $(u, X)$. Moreover, the pair $(u, X)$ defined by (4.1) must satisfy $X=X^{u}$, i.e., equation (1.1) must be fulfilled, and we want also $u$ to be $\left(\mathcal{F}_{t}^{u}\right)$-adapted. In a first step, we concentrate only on the connection $X=X^{u}$. Inserting (4.1) into (1.1), we see that we must have

or equivalently

$$
\int_{0}^{t} \mathcal{X}(t-s) \mathrm{d} B_{s}^{H}=a \int_{0}^{t}\left\{\int_{0}^{s} \mathcal{X}(s-r) \mathrm{d} B_{r}^{H}\right\} \mathrm{d} s+b \int_{0}^{t}\left\{\int_{0}^{s} \mathcal{U}(s-r) \mathrm{d} B_{r}^{H}\right\} \mathrm{d} s+B_{t}^{H},
$$

$$
\int_{0}^{t} \mathcal{X}(t-r) \mathrm{d} B_{r}^{H}=a \int_{0}^{t}\left\{\int_{r}^{t} \mathcal{X}(s-r) \mathrm{d} s\right\} \mathrm{d} B_{r}^{H}+b \int_{0}^{t}\left\{\int_{r}^{t} \mathcal{U}(s-r) \mathrm{d} s\right\} \mathrm{d} B_{r}^{H}+\int_{0}^{t} \mathrm{~d} B_{r}^{H} .
$$

This may by realized by choosing the following connection between $\mathcal{X}$ and $\mathcal{U}$

$$
\mathcal{X}(s)=a \int_{0}^{s} \mathcal{X}(\tau) \mathrm{d} \tau+b \int_{0}^{s} \mathcal{U}(\tau) \mathrm{d} \tau+1,
$$

or equivalently

$$
\dot{\mathcal{X}}(s)=a \mathcal{X}(s)+b \mathcal{U}(s) ; \quad \mathcal{X}(0)=1 .
$$

Our guess is that the minimum for $J$ can be obtained by choosing $\mathcal{U}$ in such a way that if $(\hat{u}, \hat{X})$ are defined by (4.2) with $\mathcal{X}$ governed by (4.3), then the minimum value of

$$
\hat{J}(\mathcal{U})=\mathbb{E}\left[q \hat{X}_{0}^{2}+r \hat{u}_{0}^{2}\right],
$$

\footnotetext{
${ }^{1}$ For simplicity, here we deal only with the case $x=0$.
} 
is achieved. Actually, for a stochastic integral

$$
S_{t}=\int_{-\infty}^{t} g(t-s) \mathrm{d} B_{s}^{H}
$$

we can evaluate

$$
\mathbb{E} S_{0}^{2}=H(2 H-1) \int_{0}^{+\infty} \int_{0}^{+\infty} g(s) g(r)|s-r|^{2 H-2} \mathrm{~d} s \mathrm{~d} r
$$

Exploiting the representation

$$
|s-r|^{2 H-2}=\frac{1}{B(H-1 / 2,2-2 H)} \int_{s \vee r}^{+\infty}(\tau-s)^{H-3 / 2}(\tau-r)^{H-3 / 2} \mathrm{~d} \tau,
$$

it is easy to check that we can rewrite

$$
\mathbb{E} S_{0}^{2}=\frac{2 H \Gamma\left(\frac{3}{2}-H\right)}{\Gamma\left(H+\frac{1}{2}\right) \Gamma(2-2 H)} \int_{0}^{+\infty} \widetilde{g}^{2}(s) \mathrm{d} s,
$$

where

Hence we can rewrite also

$$
\widetilde{g}(s)=\frac{\mathrm{d}}{\mathrm{d} s} \int_{0}^{s} g(r)(s-r)^{H-1 / 2} \mathrm{~d} r .
$$

$$
\hat{J}(\mathcal{U})=\widetilde{J}(\widetilde{\mathcal{U}})=\frac{2 H \Gamma\left(\frac{3}{2}-H\right)}{\Gamma\left(H+\frac{1}{2}\right) \Gamma(2-2 H)} \int_{0}^{+\infty}\left\{q \widetilde{\mathcal{X}}^{2}(s)+r \widetilde{\mathcal{U}}^{2}(s)\right\} \mathrm{d} s,
$$

where $\widetilde{\mathcal{X}}$ and $\widetilde{\mathcal{U}}$ correspond to $\mathcal{X}$ and $\mathcal{U}$ by (4.4). Actually, it is readily seen from (4.3) that the dynamics which links $\widetilde{\mathcal{X}}$ to $\tilde{\mathcal{U}}$ is nothing else but

$$
\dot{\widetilde{\mathcal{X}}}(s)=a \tilde{\mathcal{X}}(s)+b \tilde{\mathcal{U}}(s)+\left(H-\frac{1}{2}\right) s^{H-3 / 2} ; \quad \tilde{\mathcal{X}}(0)=0
$$

Applying Theorem 4.1 of [11] (see also the particular case 4.2 therein), we get the following solution of the concerned infinite time horizon deterministic control problem.

Lemma 4.1. Let the pair $\left(\widetilde{\mathcal{U}}^{*}, \widetilde{\mathcal{X}}^{*}\right)$ be governed by

$$
\begin{gathered}
\widetilde{\mathcal{U}}_{t}^{*}=-\frac{b}{r} \rho\left[\tilde{\mathcal{X}}_{t}^{*}+\widetilde{V}^{*}(t)\right], \\
\dot{\tilde{\mathcal{X}}}^{*}(s)=a \widetilde{\mathcal{X}}^{*}(s)+b \widetilde{\mathcal{U}}^{*}(s)+\left(H-\frac{1}{2}\right) s^{H-3 / 2} ; \quad \widetilde{\mathcal{X}}^{*}(0)=0,
\end{gathered}
$$

where

$$
\widetilde{V}^{*}(\tau)=\left(H-\frac{1}{2}\right) \int_{0}^{+\infty} \mathrm{e}^{-\delta r}(\tau+r)^{H-3 / 2} \mathrm{~d} r,
$$

with $(\rho, \delta)$ given by (1.4). Then, for $\widetilde{J}$ defined by $(4.5)$, the pair $\left(\widetilde{\mathcal{U}}^{*}, \widetilde{\mathcal{X}}^{*}\right)$ is optimal in the control problem

$$
\min _{\widetilde{\mathcal{U}}} \widetilde{J}(\widetilde{\mathcal{U}}) \quad \text { subject to }(4.6) \text {. }
$$

Moreover, the value of the optimal cost is $\widetilde{J}\left(\widetilde{\mathcal{U}}^{*}\right)=\bar{\lambda}$ where $\bar{\lambda}$ is given by (3.9). 
Now, taking into account the fact that the connection (4.4) can be inverted as

$$
g(s)=\frac{1}{B\left(H+\frac{1}{2}, \frac{3}{2}-H\right)} \frac{\mathrm{d}}{\mathrm{d} s} \int_{0}^{s} \widetilde{g}(r)(s-r)^{1 / 2-H} \mathrm{~d} r
$$

we may reformulate our initial guess by telling that the pair $\left(\mathcal{U}^{*}, \mathcal{X}^{*}\right)$ obtained through $(4.10)$ from $\left(\widetilde{\mathcal{U}}^{*}, \widetilde{\mathcal{X}}^{*}\right)$ is a candidate to define through (4.1) an optimal pair $\left(u^{*}, X^{*}\right)$ in the infinite time horizon stochastic control problem. Actually, this is true and the proof below of the following statement includes the proof that it is.

Theorem 4.1. Let the pair $\left(u^{*}, X^{*}\right)$ be governed by

$$
\begin{gathered}
u_{t}^{*}=-\frac{b}{r} \rho\left[X_{t}^{*}+v_{t}^{*}\right] ; \quad X_{t}^{*}=X_{t}^{u^{*}} \\
v_{t}^{*}=\int_{0}^{t} \delta v_{s}^{*} \mathrm{~d} s+\int_{0}^{t}\left[\frac{\delta^{\frac{1}{2}-H}}{\Gamma\left(\frac{3}{2}-H\right)}(t-s)^{\frac{1}{2}-H}-1\right]\left\{\mathrm{d} X_{s}^{*}-\left[a X_{s}^{*}+b u_{s}^{*}\right] \mathrm{d} s\right\}
\end{gathered}
$$

where $(\rho, \delta)$ is given by (1.4). Then, for $J$ defined by (1.2), the pair $\left(u^{*}, X^{*}\right)$ is optimal in the control problem

$$
\min _{u \in \mathcal{U}_{a d}} J(u) \quad \text { subject to }(1.1)
$$

i.e., $J\left(u^{*}\right)=\bar{\lambda}$ a.s. where $\bar{\lambda}$ is given by (3.9).

Proof. Due to the discussion above, we start with the pair $\left(u^{*}, X^{*}\right)$ defined by

$$
u_{t}^{*}=\int_{0}^{t} \mathcal{U}^{*}(t-s) \mathrm{d} B_{s}^{H} ; \quad X_{t}^{*}=\int_{0}^{t} \mathcal{X}^{*}(t-s) \mathrm{d} B_{s}^{H},
$$

where

$$
\begin{aligned}
& \mathcal{U}^{*}(s)=\frac{1}{B\left(H+\frac{1}{2}, \frac{3}{2}-H\right)} \frac{\mathrm{d}}{\mathrm{d} s} \int_{0}^{s} \widetilde{\mathcal{U}}^{*}(r)(s-r)^{1 / 2-H} \mathrm{~d} r \\
& \mathcal{X}^{*}(s)=\frac{1}{B\left(H+\frac{1}{2}, \frac{3}{2}-H\right)} \frac{\mathrm{d}}{\mathrm{d} s} \int_{0}^{s} \widetilde{\mathcal{X}}^{*}(r)(s-r)^{1 / 2-H} \mathrm{~d} r
\end{aligned}
$$

with $\left(\widetilde{\mathcal{U}}^{*}, \widetilde{\mathcal{X}}^{*}\right)$ governed by the system $(4.7)-(4.8)$. It is easy to check that actually

$$
\begin{aligned}
& \mathcal{U}^{*}(s)=-\frac{b}{r} \rho\left[\mathcal{X}^{*}(s)+V^{*}(s)\right], \\
& \dot{\mathcal{X}}^{*}(s)=a \mathcal{X}^{*}(s)+b \mathcal{U}^{*}(s) ; \quad \mathcal{X}^{*}(0)=1,
\end{aligned}
$$

where $V^{*}$, which corresponds through (4.10) to $\widetilde{V}^{*}$ given by (4.9), is defined by

$$
V^{*}(t)=\frac{H-\frac{1}{2}}{B\left(H+\frac{1}{2}, \frac{3}{2}-H\right)} \int_{0}^{+\infty} \mathrm{e}^{-\delta t r} \frac{r^{H-1 / 2}}{r+1} \mathrm{~d} r .
$$

Then, introducing the process

$$
v_{t}^{*}=\int_{0}^{t} V^{*}(t-s) \mathrm{d} B_{s}, \quad t \geq 0,
$$

from definitions (4.13), (4.16) and equations (4.14), (4.15), one can parallel the proof of Theorem 3.1 in [11] (see also Rem. 3.1 therein) in order to show that the triple $\left(u^{*}, X^{*}, v^{*}\right)$ is governed by the system (4.11)-(4.12). 
Now, it remains to verify that the pair $\left(u^{*}, X^{*}\right)$ is optimal, i.e., that with probability one

$$
\lim _{T \rightarrow+\infty} \frac{1}{T} \int_{0}^{T}\left[q\left(X_{t}^{*}\right)^{2}+r\left(u_{t}^{*}\right)^{2}\right] \mathrm{d} t=\lim _{T \rightarrow+\infty} \frac{1}{T} \int_{0}^{T}\left[q\left(\bar{X}_{t}\right)^{2}+r\left(\bar{u}_{t}\right)^{2}\right] \mathrm{d} t
$$

where $(\bar{u}, \bar{X})$ is the optimal pair given by (3.6)-(3.7). Clearly, this will hold if we are able to prove that

$$
\lim _{t \rightarrow+\infty}\left(X_{t}^{*}-\bar{X}_{t}\right)=0 \text { a.s.; } \lim _{t \rightarrow+\infty}\left(u_{t}^{*}-\bar{u}_{t}\right)=0 \text { a.s. }
$$

But, since the pairs $\left(u^{*}, X^{*}\right)$ and $(\bar{u}, \bar{X})$ satisfy equations (3.6) and (4.11) respectively, it is readily seen that

$$
\mathrm{d}\left(X_{t}^{*}-\bar{X}_{t}\right)=-\delta\left(X_{t}^{*}-\bar{X}_{t}\right) \mathrm{d} t-\frac{b^{2}}{r} \rho\left(v_{t}^{*}-\bar{v}_{t}\right) \mathrm{d} t ; \quad X_{0}^{*}-\bar{X}_{0}=0
$$

where $v^{*}$ and $\bar{v}$ are given by (3.7) and (4.12) respectively. Hence, to prove (4.17), it is sufficient to show that

$$
\lim _{t \rightarrow+\infty}\left(\bar{v}_{t}-v_{t}^{*}\right)=0 \text { a.s. }
$$

From Remarks 3.1 and 3.2, we know that

$$
\bar{v}_{t}=\mathbb{E}\left(\xi_{t} / \mathcal{F}_{t}^{H}\right)
$$

where $\xi$ is the stationary process defined by

$$
\xi_{t}=\int_{t}^{+\infty} \mathrm{e}^{-\delta(s-t)} \mathrm{d} B_{s}^{H}, \quad t \geq 0
$$

Similarly, it can be checked that defining the process $\hat{v}$ by

$$
\hat{v}_{t}=\int_{-\infty}^{t} V^{*}(t-s) \mathrm{d} B_{s}^{H}, \quad t \geq 0
$$

the variable $\hat{v}_{t}$ can be interpreted as

$$
\hat{v}_{t}=\mathbb{E}\left(\xi_{t} / \mathcal{F}_{-\infty, t}^{H}\right)
$$

where $\left(\mathcal{F}_{-\infty, t}^{H} ; t \in \mathbb{R}\right)$ is the natural filtration of the two-sided fractional Brownian motion $B^{H}$. Indeed, using (4.15), from (4.18) we get that

$$
\begin{aligned}
\hat{v}_{t} & =\frac{H-\frac{1}{2}}{B\left(H+\frac{1}{2}, \frac{3}{2}-H\right)} \mathrm{e}^{\delta t} \int_{-\infty}^{t}(t-s)^{\frac{1}{2}-H} \int_{t}^{+\infty} \mathrm{e}^{-\delta \tau} \frac{(\tau-t)^{H-\frac{1}{2}}}{\tau-s} \mathrm{~d} \tau \mathrm{d} B_{s}^{H} \\
& =\frac{1}{B\left(H-\frac{1}{2}, \frac{3}{2}-H\right)} \int_{t}^{+\infty} \mathrm{e}^{\delta(t-\tau)} \int_{-\infty}^{t} \frac{(t-s)^{\frac{1}{2}-H}(\tau-t)^{H-\frac{1}{2}}}{\tau-s} \mathrm{~d} B_{s}^{H} \mathrm{~d} \tau \\
& =\int_{t}^{+\infty} \mathrm{e}^{\delta(t-\tau)} \mathrm{d} \mathbb{E}\left(B_{\tau}^{H} / \mathcal{F}_{-\infty, t}^{H}\right),
\end{aligned}
$$

where the last equality holds thanks to the representation of the predictor $\mathbb{E}\left(B_{\tau}^{H} / \mathcal{F}_{-\infty, t}^{H}\right)$ which is derived in [15] (see expression (14) therein).

From (4.19), since $v_{t}^{*}$ is $\mathcal{F}_{t}^{H}$-measurable, we can write

$$
\bar{v}_{t}-v_{t}^{*}=\mathbb{E}\left(\left[\hat{v}_{t}-v_{t}^{*}\right] / \mathcal{F}_{t}^{H}\right), \quad t \geq 0
$$


Consequently, thanks to Proposition 5.3 of the Appendix, to prove that

$$
\lim _{t \rightarrow+\infty}\left(\bar{v}_{t}-v_{t}^{*}\right)=0 \quad \text { a.s. }
$$

it is sufficient to check that

$$
\lim _{t \rightarrow+\infty}\left(\hat{v}_{t}-v_{t}^{*}\right)=0 \quad \text { a.s. }
$$

But, due to (4.16) and (4.18), we have

$$
\hat{v}_{t}-v_{t}^{*}=\int_{-\infty}^{0} V^{*}(t-s) \mathrm{d} B_{s}^{H}, \quad t \geq 0
$$

which, integrating by parts, gives

$$
\hat{v}_{t}-v_{t}^{*}=\int_{-\infty}^{0} \dot{V}^{*}(t-s) B_{s}^{H} \mathrm{~d} s
$$

where $\dot{V}^{*}(\tau)$ stands for the derivative of $V^{*}(\tau)$ with respect to $\tau$. Hence, we have

$$
\left|\hat{v}_{t}-v_{t}^{*}\right| \leq \int_{-\infty}^{0}\left|\dot{V}^{*}(t-s)\right|\left|B_{s}^{H}\right| \mathrm{d} s,
$$

where, from the definition (4.15), one can check that the following properties hold:

(i) for all fixed $s<0,\left|\dot{V}^{*}(.-s)\right|$ is decreasing on $[0,+\infty)$;

(ii) for all fixed $s<0, \lim _{t \rightarrow+\infty}\left|\dot{V}^{*}(t-s)\right|\left|B_{s}^{H}\right|=0$ a.s.;

(iii) for all fixed $t>0, \int_{-\infty}^{0}\left|\dot{V}^{*}(t-s)\right|\left|B_{s}^{H}\right| \mathrm{d} s<\infty$ a.s.

So we can apply the theorem of dominated convergence to get that a.s.

$$
\lim _{t \rightarrow+\infty} \int_{-\infty}^{0}\left|\dot{V}^{*}(t-s)\right|\left|B_{s}^{H}\right| \mathrm{d} s=\int_{-\infty}^{0}\left[\lim _{t \rightarrow+\infty}\left|\dot{V}^{*}(t-s)\right|\left|B_{s}^{H}\right|\right] \mathrm{d} s=0
$$

which achieves the proof of the theorem.

Remark 4.1. It is worth to emphasize that, due to the non Markovian setting, it is not surprising that the optimal policy $u^{*}$ is not an instantaneous feedback control. Nevertheless, we can try to find an optimal policy in the subclass of such admissible controls of the form $u_{t}=\mu X_{t}$, where $X=X^{u}$ and $\mu$ is some fixed coefficient. Then the state process $X$ is governed by the equation

$$
\mathrm{d} X_{t}=(a+b \mu) X_{t} \mathrm{~d} t+\mathrm{d} B_{t}^{H}, X_{0}=x,
$$

and the performance criterion $J(u)$ to minimize becomes $J^{*}(\mu)$ where

$$
J^{*}(\mu)=\left(q+r \mu^{2}\right) \limsup _{T \rightarrow+\infty} \frac{1}{T} \int_{0}^{T} X_{t}^{2} \mathrm{~d} t .
$$

It can be checked that if $a+b \mu \geq 0$ then $J^{*}(\mu)=+\infty$ a.s. and so we concentrate on values of $\mu$ such that $a+b \mu<0$. Then the process $X$ is an asymptotically stationary ergodic process and

$$
J^{*}(\mu)=\left(q+r \mu^{2}\right) \lim _{T \rightarrow+\infty} \mathbb{E} X_{T}^{2} \quad \text { a.s. },
$$

where

$$
\lim _{T \rightarrow+\infty} \mathbb{E} X_{T}^{2}=\frac{\Gamma(2 H+1)}{2|a+b \mu|^{2 H}}
$$


It is readily seen that to minimize $J^{*}(\mu)$ with respect to $\mu$, one must take $\mu=\mu^{*}$ where

$$
\mu^{*}=-\frac{a+\sqrt{a^{2}+4 \frac{b^{2}}{r} q H(1-H)}}{2 b(1-H)}
$$

which leads to the cost function value

$$
J^{*}\left(\mu^{*}\right)=\Gamma(2 H) \frac{r\left|\mu^{*}\right|}{b}\left|a+b \mu^{*}\right|^{1-2 H} .
$$

For example, if $a=0$ and $b=q=r=1$, we get

$$
\mu^{*}=-\sqrt{\frac{H}{1-H}} ; \quad J^{*}\left(\mu^{*}\right)=\Gamma(2 H)\left(\frac{H}{1-H}\right)^{1-H} .
$$

Actually, under the same conditions, in the original problem, the optimal cost $J(\bar{u})=J\left(u^{*}\right)=\bar{\lambda}$ given by $(3.9)$ is

$$
J(\bar{u})=\Gamma(2 H) H(1+\sin \pi H) .
$$

\section{Appendix}

Here we prove auxiliary results around ergodic properties of some processes. At first, we analyze the asymptotic behaviour of a specific Gaussian process.

Proposition 5.1. Let $\gamma(t, t)$ be defined by (3.1) for $s=t$, i.e.,

$$
\gamma(t, t)=\delta \mathrm{e}^{\delta t} \int_{t}^{+\infty} \mathrm{e}^{-\delta r} K_{H}(r, t) \mathrm{d} r
$$

with $K_{H}$ given by (3.2) and let $\left(\bar{w}_{t}, t \geq 0\right)$ be the process defined by (3.24), i.e., the solution of the stochastic differential equation

$$
\mathrm{d} \bar{w}_{t}=-\delta \bar{w}_{t} \mathrm{~d} t+\gamma(t, t) \mathrm{d} M_{t}^{H} ; \quad \bar{w}_{0}=x,
$$

where $M^{H}$ is the Gaussian martingale defined by (2.4), with the variance function $\left\langle M^{H}\right\rangle=w^{H}$ given by (2.3). Then, the following properties hold:

(i)

$(i i)$

$$
\lim _{T \rightarrow+\infty} T^{\frac{1}{2}-H} \gamma(T, T)=\frac{2 H \Gamma\left(H+\frac{1}{2}\right)}{\delta^{H-\frac{1}{2}}}
$$

$$
\lim _{T \rightarrow+\infty} \frac{\bar{w}_{T}}{\sqrt{T}}=0 \quad \text { a.s. }
$$

(iii)

$$
\lim _{T \rightarrow+\infty} \frac{1}{T} \int_{0}^{T} \bar{w}_{t}^{2} \mathrm{~d} t=\frac{\Gamma(2 H+1)}{2 \delta^{2 H}} \sin \pi H \quad \text { a.s. }
$$

Proof. To prove assertion $(i)$, at first we observe that

$$
\gamma(t, t)=\mathrm{e}^{\delta t} \int_{t}^{\infty} \mathrm{e}^{-\delta r} \dot{K}_{H}(r, t) \mathrm{d} r
$$


where $\dot{K}_{H}$ is given by (3.21). So, by successive changes of the variable in the integrals, we can write

$$
\begin{aligned}
\gamma(t, t) & =H(2 H-1) \int_{0}^{\infty} \mathrm{e}^{-\delta u} u^{H-\frac{3}{2}}(u+t)^{H-\frac{1}{2}} \mathrm{~d} u \\
& =H(2 H-1) \int_{0}^{\infty} \mathrm{e}^{-\delta t \tau} \tau^{H-\frac{3}{2}}(1+\tau)^{H-\frac{1}{2}} \mathrm{~d} \tau .
\end{aligned}
$$

Hence, using the Laplace approximation method, we get immediately that

$$
\begin{aligned}
\lim _{t \rightarrow+\infty} t^{\frac{1}{2}-H} \gamma(t, t) & =\lim _{t \rightarrow+\infty} H(2 H-1) t^{\frac{1}{2}-H} \int_{0}^{\infty} \mathrm{e}^{-\delta t \tau} \tau^{H-\frac{3}{2}} \mathrm{~d} \tau \\
& =H(2 H-1) \Gamma\left(H-\frac{1}{2}\right) \delta^{\frac{1}{2}-H}=\frac{2 H \Gamma\left(H+\frac{1}{2}\right)}{\delta^{H-\frac{1}{2}}},
\end{aligned}
$$

which means exactly that assertion $(i)$ holds.

Now, to prove assertions $(i i)$ and (iii), we parallel the proof of Lemma 6 in [12]. Actually, due to the representation (3.24), i.e.,

$$
\bar{w}_{T}=\mathrm{e}^{-\delta T}\left\{x+\int_{0}^{T} \mathrm{e}^{\delta s} \gamma(s, s) \mathrm{d} M_{s}^{H}\right\},
$$

exploiting the limiting property $(i)$, assertion (ii) follows directly from the law of iterated logarithm for continuous martingales.

To prove assertion $(i i i)$, at first we apply the Itô formula to the process $\left(\bar{w}_{t}^{2}, t \in[0, T]\right)$ to get the representation

$$
\bar{w}_{T}^{2}-\bar{w}_{0}^{2}=-2 \delta \int_{0}^{T} \bar{w}_{t}^{2} \mathrm{~d} t+2 \int_{0}^{T} \gamma(t, t) \bar{w}_{t} \mathrm{~d} M_{t}^{H}+\int_{0}^{T} \gamma^{2}(t, t) \mathrm{d}\left\langle M^{H}\right\rangle_{t} .
$$

This can be rewritten as

$$
\frac{1}{T} \int_{0}^{T} \bar{w}_{t}^{2} \mathrm{~d} t\left[1-\frac{L_{T}}{\langle L\rangle_{T}} \Psi_{T}\right]=\frac{1}{2 \delta T}\left[x^{2}-\bar{w}_{T}^{2}\right]+\frac{1}{2 \delta T} \int_{0}^{T} \gamma^{2}(t, t) \mathrm{d}\left\langle M^{H}\right\rangle_{t}
$$

where

$$
L_{T}=\int_{0}^{T} \gamma(t, t) \bar{w}_{t} \mathrm{~d} M_{t}^{H} ; \quad\langle L\rangle_{T}=\int_{0}^{T} \gamma^{2}(t, t) \bar{w}_{t}^{2} \mathrm{~d}\left\langle M^{H}\right\rangle_{t} ; \quad \Psi_{T}=\frac{\langle L\rangle_{T}}{\delta \int_{0}^{T} \bar{w}_{t}^{2} \mathrm{~d} t} .
$$

Since $\left\langle M^{H}\right\rangle$ is nothing but the function $w^{H}$ given by $(2.3)$, thanks to $(i)$, we get that

$$
\lim _{T \rightarrow+\infty} \frac{1}{2 \delta T} \int_{0}^{T} \gamma^{2}(t, t) \mathrm{d}\left\langle M^{H}\right\rangle_{t}=\frac{\Gamma(2 H+1)}{2 \delta^{2 H}} \sin \pi H
$$

Hence, exploiting (ii), we see that the right hand side in (5.1) tends to the same limit. Now, in the left hand side of (5.1), we observe that due to assertion $(i)$, we have

$$
0<\lim _{T \rightarrow+\infty} \Psi_{T}<\infty \quad \text { a.s. }
$$

Therefore, making use of Lemma 2.6.3 in [14] to discuss the behaviour of the factor within brackets, it is readily seen that the statement (iii) holds. 
Now, we analyze the asymptotic behaviour of some stationary continuous processes.

Proposition 5.2. Let $\left(G_{t}, t \in \mathbb{R}\right)$ be a stationary continuous process. Then, the following assertions hold:

(i) if

then

$$
\mathbb{E} \sup _{t \in[0,1]}\left|G_{t}\right|^{2}<+\infty
$$

$$
\lim _{T \rightarrow+\infty} \frac{G_{T}}{\sqrt{T}}=0 \quad \text { a.s. }
$$

(ii) if $\left(G_{t}, t \in \mathbb{R}\right)$ is Gaussian, then the condition (5.2) is fulfilled and (5.3) holds.

Proof. To prove assertion $(i)$, we must show that if $(5.2)$ is fulfilled, then for all $l \in \mathbb{N}^{*}$

$$
\mathbb{P}\left\{\limsup _{T \rightarrow+\infty} \frac{\left|G_{T}\right|}{\sqrt{T}}>\frac{1}{l}\right\}=0 .
$$

But, clearly we have

$$
\begin{aligned}
\mathbb{P}\left\{\limsup _{T \rightarrow+\infty} \frac{\left|G_{T}\right|}{\sqrt{T}}>\frac{1}{l}\right\} & =\mathbb{P}\left\{\cap_{n \in \mathbb{N}} \cup_{m \geq n}\left\{\sup _{t \in[m, m+1]} \frac{\left|G_{t}\right|}{\sqrt{t}}>\frac{1}{l}\right\}\right\} \\
& \leq \mathbb{P}\left\{\cap_{n \in \mathbb{N}} \cup_{m \geq n}\left\{\sup _{t \in[m, m+1]}\left|G_{t}\right|>\frac{\sqrt{m}}{l}\right\}\right\} \\
& =\lim _{n \rightarrow+\infty} \mathbb{P}\left\{\cup_{m \geq n}\left\{\sup _{t \in[m, m+1]}\left|G_{t}\right|>\frac{\sqrt{m}}{l}\right\}\right\} \\
& \leq \lim _{n \rightarrow+\infty} \sum_{m=n}^{+\infty} \mathbb{P}\left\{\sup _{t \in[m, m+1]}\left|G_{t}\right|>\frac{\sqrt{m}}{l}\right\} .
\end{aligned}
$$

So, due to the stationarity assumption, we get that

$$
\begin{aligned}
\mathbb{P}\left\{\limsup _{T \rightarrow+\infty} \frac{\left|G_{T}\right|}{\sqrt{T}}>\frac{1}{l}\right\} & \leq \lim _{n \rightarrow+\infty} \sum_{m=n}^{+\infty} \mathbb{P}\left\{\sup _{t \in[0,1]}\left|G_{t}\right|>\frac{\sqrt{m}}{l}\right\} \\
& =\lim _{n \rightarrow+\infty} \sum_{m=n}^{+\infty} \mathbb{P}\left\{\eta_{l}>m\right\}
\end{aligned}
$$

where $\eta_{l}=l^{2} \sup _{t \in[0,1]}\left|G_{t}\right|^{2}$. But condition (5.2) implies that $\mathbb{E} \eta_{l}<+\infty$ and so, for all $l \in \mathbb{N}^{*}$, we have

$$
\lim _{n \rightarrow+\infty} \sum_{m=n}^{\infty} \mathbb{P}\left\{\eta_{l}>m\right\}=0 .
$$

This gives that (5.3) holds, which means that the statement $(i)$ is valid.

To prove assertion $(i i)$, at first we observe that, due to the continuity of the process $\left(G_{t}, t \in \mathbb{R}\right)$, we have

$$
\sup _{t \in[0,1]}\left|G_{t}\right|=\sup _{q \in[0,1] \cap \mathbb{Q}}\left|G_{q}\right|<+\infty \quad \text { a.s. }
$$

Then, defining the function

$$
N_{1}(x)=\sup _{q \in[0,1] \cap \mathbb{Q}}\left|x_{q}\right| ; \quad x=\left(x_{q}, q \in[0,1] \cap \mathbb{Q}\right)
$$




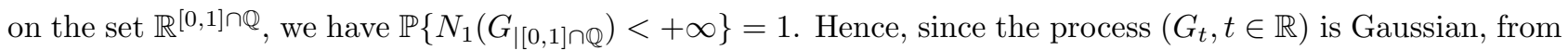
the corollary of Lemma 4.9.2 in [14], we get that

$$
\mathbb{E} N_{1}^{2}\left(G_{\mid[0,1] \cap \mathbb{Q}}\right)<+\infty
$$

So, it turns that the condition (5.2) is fulfilled.

Finally, we prove a Levy type result for Gaussian processes.

Proposition 5.3. Let $\left(G_{t}\right), t \in \mathbb{R}$ be a continuous Gaussian process such that

$$
\lim _{T \rightarrow+\infty} G_{T}=0 \quad \text { a.s. }
$$

Then, for all family $\left(\mathcal{F}_{t}, t \in \mathbb{R}\right)$ of nondecreasing $\sigma$-algebras, the following property holds:

$$
\lim _{T \rightarrow+\infty} \mathbb{E}\left(G_{T} / \mathcal{F}_{T}\right)=0 \quad \text { a.s. }
$$

Proof. We need to show that for any sequence $\tau=\left\{t_{n}, n \in \mathbb{N}\right\}$ which tends to infinity as $n$ goes to infinity, we have

$$
\lim _{n \rightarrow+\infty} \mathbb{E}\left(G_{t_{n}} / \mathcal{F}_{t_{n}}\right)=0 \quad \text { a.s. }
$$

To prove this, according to the Theorem 1.6 in [13] (see also [2]), it is sufficient to check that

$$
\mathbb{E}\left(\sup _{n \in \mathbb{N}}\left|G_{t_{n}}\right|\right)<+\infty
$$

We define the function

$$
N_{2}(x)=\sup _{n \in \mathbb{N}}\left|x_{t_{n}}\right| ; \quad x=\left(x_{t_{n}}, n \in \mathbb{N}\right),
$$

on the set $\mathbb{R}^{\tau}$. Since thanks to (5.4) we have $\lim _{n \rightarrow+\infty} G_{t_{n}}=0$ a.s., it turns that $\mathbb{P}\left\{N_{2}\left(G_{\mid \tau}\right)<+\infty\right\}=1$. Hence, since the process $\left(G_{t}, t \in \mathbb{R}\right)$ is Gaussian, from the corollary of Lemma 4.9.2 in [14], we get that

$$
\mathbb{E} N_{2}\left(G_{\mid \tau}\right)<+\infty
$$

which means exactly that the condition (5.5) is fulfilled.

\section{REFERENCES}

[1] F. Biaggini, Y. Hu, B. Øksendal and A. Sulem, A stochastic maximum principle for processes driven by fractional Brownian motion. Stochastic Processes Appl. 100 (2002) 233-253.

[2] D. Blackwell and L. Dubins, Merging of opinions with increasing information. Ann. Math. Statist. 33 (1962) $882-886$.

[3] M.H.A. Davis, Linear Estimation and Stochastic Control. Chapman and Hall, New York (1977).

[4] L. Decreusefond and A.S. Üstünel, Stochastic analysis of the fractional Brownian motion. Potential Anal. 10 (1999) $177-214$.

[5] T.E. Duncan, Y. Hu and B. Pasik-Duncan, Stochastic calculus for fractional Brownian motion I. Theory. SIAM J. Control Optim. 38 (2000) 582-612.

[6] G. Gripenberg and I. Norros, On the prediction of fractional Brownian motion. J. Appl. Probab. 33 (1996) 400-410.

[7] M.L. Kleptsyna and A. Le Breton, Statistical analysis of the fractional Ornstein-Uhlenbeck type process. Statist. Inference Stochastic Processes 5 (2002) 229-248.

[8] M.L. Kleptsyna and A. Le Breton, Extension of the Kalman-Bucy filter to elementary linear systems with fractional Brownian noises. Statist. Inference Stochastic Processes 5 (2002) 249-271.

[9] M.L. Kleptsyna, A. Le Breton and M.-C. Roubaud, General approach to filtering with fractional Brownian noises - Application to linear systems. Stochastics Reports 71 (2000) 119-140.

[10] M.L. Kleptsyna, A. Le Breton and M. Viot, About the linear-quadratic regulator problem under a fractional Brownian perturbation. ESAIM: PS 7 (2003) 161-170. 
[11] M.L. Kleptsyna, A. Le Breton and M. Viot, Asymptotically optimal filtering in linear systems with fractional Brownian noises. Statist. Oper. Res. Trans. (2004) 28 177-190.

[12] A. Le Breton, Adaptive control in the scalar linear-quadratic model in continious time. Statist. Probab. Lett. 13 (1992) $169-177$.

[13] R.S. Liptser and A.N. Shiryaev, Statist. Random Processes. Springer-Verlag, New York (1978).

[14] R.S. Liptser and A.N. Shiryaev, Theory of Martingales. Kluwer Academic Publ., Dordrecht (1989).

[15] G.M. Molchan, Linear problems for fractional Brownian motion: group approach. Probab. Theory Appl. 1 (2002) 59-70 (in Russian).

[16] G.M. Molchan, Gaussian processes with spectra which are asymptotically equivalent to a power of $\lambda$. Probab. Theory Appl. 14 (1969) 530-532.

[17] G.M. Molchan and J.I. Golosov, Gaussian stationary processes with which are asymptotic power spectrum. Soviet Math. Dokl. 10 (1969) 134-137.

[18] I. Norros, E. Valkeila and J. Virtamo, An elementary approach to a Girsanov formula and other analytical results on fractional Brownian motions. Bernoulli 5 (1999) 571-587.

[19] C.J. Nuzman and H.V. Poor, Linear estimation of self-similar processes via Lamperti's transformation. J. Appl. Prob. 37 (2000) 429-452. 